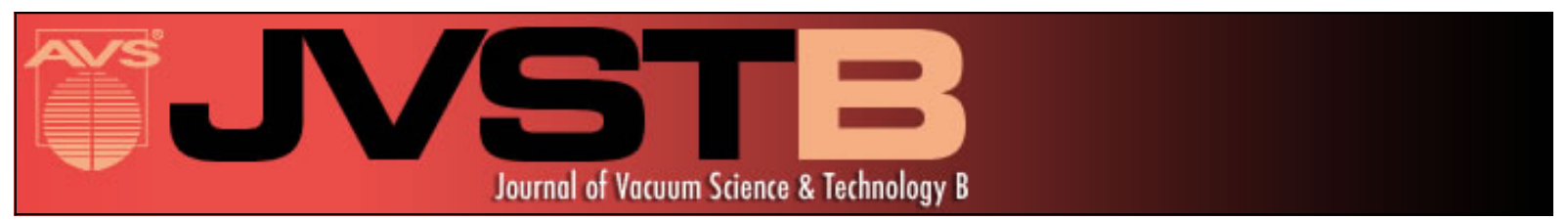

\title{
Fiber tip-based electron source
}

Albert Casandruc, Günther Kassier, Haider Zia, Robert Bücker, and R. J. Dwayne Miller

Citation: Journal of Vacuum Science \& Technology B 33, 03C101 (2015); doi: 10.1116/1.4902016

View online: http://dx.doi.org/10.1116/1.4902016

View Table of Contents: http://scitation.aip.org/content/avs/journal/jvstb/33/3?ver=pdfcov

Published by the AVS: Science \& Technology of Materials, Interfaces, and Processing

\section{Articles you may be interested in}

Tip-based source of femtosecond electron pulses at $30 \mathrm{keV}$

J. Appl. Phys. 115, 094506 (2014); 10.1063/1.4867185

Source of low-energy coherent electron beams

Appl. Phys. Lett. 88, 023101 (2006); 10.1063/1.2161942

Emission properties of a dual ion/electron source based on Au-In alloy

Appl. Phys. Lett. 80, 1480 (2002); 10.1063/1.1453484

Micromachined ultrasharp silicon and diamond-coated silicon tip as a stable field-emission electron source and a scanning probe microscopy sensor with atomic sharpness

J. Vac. Sci. Technol. B 16, 3185 (1998); 10.1116/1.590348

Nanostructured integrated electron source

J. Vac. Sci. Technol. B 16, 862 (1998); 10.1116/1.589921

\section{HDDEN}

\section{Instruments for Advanced Science}

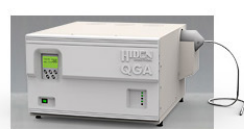

Gas Analysis

- dynamic measurement of reaction gas streams catalysis and thermal analysis

molecular beam studies

- dissolved species probes

fermentation, environmental and ecological studies

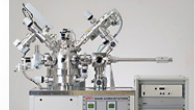

Surface Science

, UHVTPD

SIMS elemental imaging - surface mapoing

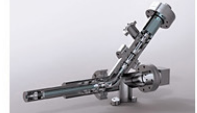

Plasma Diagnostics plasma source characterization etch and deposition process reaction kinetic studies , analysis of neutral and radical species

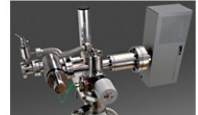

Vacuum Analysis ' partial pressure measurement and control of process gases reactive sputter process contro , vacuum diagnostics vacuum coating process monitoring 


\title{
Fiber tip-based electron source
}

\author{
Albert Casandruc, Günther Kassier, Haider Zia, and Robert Bücker \\ Max Planck Institute for the Structure and Dynamics of Matter, CFEL (Bld. 99), Luruper Chaussee 149, \\ 22761 Hamburg, Germany \\ R. J. Dwayne Miller ${ }^{\text {a) }}$ \\ Max Planck Institute for the Structure and Dynamics of Matter, CFEL (Bld. 99), Luruper Chaussee 149, \\ 22761 Hamburg, Germany and Departments of Chemistry and Physics, University of Toronto, Toronto, \\ Ontario M5S 3H6, Canada
}

(Received 29 August 2014; accepted 30 October 2014; published 26 November 2014)

\begin{abstract}
The authors report on the first experimental characterization of a fiber tip-based electron source, where electron emission can be triggered by both electric field and optical excitation. Our approach consists of coating the open aperture of a commercial $100 \mathrm{~nm}$ apex size near-field scanning optical microscopy fiber tip with a $10 \mathrm{~nm}$ thick tungsten (W) layer, which is back-illuminated by a $405 \mathrm{~nm}$ continuous-wave laser beam in the presence of an extraction electric field. Despite the very low optical transmission of the fiber due to the subwavelength aperture size, measurements show a clearly enhanced emission when photoexciting the $\mathrm{W}$ layer with respect to pure field emission. The emission response time is slower than the optical trigger time, suggesting that thermal effects are predominant in the studied regime. To back up this hypothesis, the authors fabricated a nanometric thermocouple probe based on a Pt/Au junction and measured the temporal response of the tip temperature. The measured switch-on time for the tip temperature is consistent with the switch-on time of the optically enhanced electron emission. (C) 2014 American Vacuum Society.

[http://dx.doi.org/10.1116/1.4902016]
\end{abstract}

\section{INTRODUCTION}

Pulsed high brightness electron sources have found wide application in time-resolved electron diffraction ${ }^{1-3}$ and microscopy. ${ }^{4-6}$ Also, they are essential components of synchrotron and free electron laser $\mathrm{x}$-ray facilities. ${ }^{7,8}$ While planar photocathodes and laser-triggered thermionic sources are commonly employed in time-resolved experiments, ${ }^{1-6,9}$ recently interest has grown in nanometric photofield emission tip sources. ${ }^{10-19}$ Despite offering a lower peak current as compared to planar photocathodes, their small size allows for emission of extremely low-emittance and high-brightness electron bunches. The resulting large transverse coherence length at the sample plane makes them especially promising candidates for a new generation of experiments tailored to crystalline specimens with large unit cell sizes, such as proteins. ${ }^{20}$ In addition, the Schottky lowering of the emitter's work function facilitates single or few-photon photofield emission at wavelengths in the visible and near-infrared ranges. To enhance the available peak currents and provide integrated electron gun designs, tip sources can be combined into Spindt-type gated emitter arrays, ${ }^{21-24}$ also providing the option of subnanosecond electronic gating. ${ }^{25}$ While continuous field-emitter electron guns are now standard for static electron microscopy and crystallography applications, there is still much scope for the development of their phototriggered pulsed analogues, ${ }^{14,25}$ particularly regarding the difficulty of incorporating and aligning optical elements in high electric field environments.

In this paper, we present a novel approach to deliver optical radiation to a nanotip source. The method consists of

${ }^{a)}$ Electronic mail: dwayne.miller@mpsd.mpg.de applying a thin metal coating to the nanometer-sized apex of a tapered optical fiber, which acts as a source for fieldemitted electrons. This amalgamation of the electron emitter with the optical trigger source eliminates alignment issues of optical elements, while incorporating all of the advantages of back-illuminated planar photocathodes. Such a device promises to provide an electron source that is both more stable and more versatile than conventional ones based on externally triggered field emitters or flat photocathodes.

\section{DESCRIPTION OF THE DEVICE AND EXPERIMENTAL SETUP}

We use a standard near-field scanning optical microscopy (NSOM) multimode optical fiber tip ${ }^{26}$ purchased from Nanonics Imaging Ltd. The devices are compatible with photon wavelengths in the $200-1200 \mathrm{~nm}$ range and are tapered down at one end such that the fiber core forms a $100 \mathrm{~nm}$ diameter tip as shown in Fig. 1. Using a pulled fiber tip, instead of an etched one, ${ }^{27}$ assures better transmission of the light to the apex, as the cladding is not removed in the process. The tapered region features a $\mathrm{Cr} / \mathrm{Au}$ coating, providing both electrical conduction to the tip, and reducing optical losses. The $100 \mathrm{~nm}$ fiber core tip is left as an open subwavelength aperture, where only a tiny fraction of the incident light is escaping into a propagating beam. Our approach consists of covering the open aperture with a thin metallic layer, thus forming a novel nanometric electron source, which holds the advantages of sharp field emitters, but could also be used as a back-illuminated photocathode in which the difficulties of aligning optical elements are absent. The coatings were done using an electron-beam deposition machine, where the fibers were mounted under an angle of 


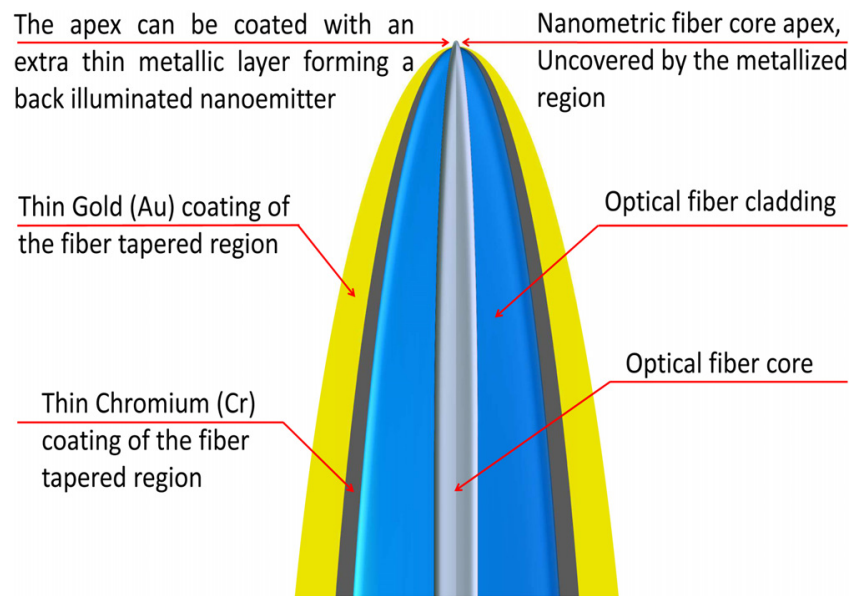

FIG. 1. (Color online) Schematic view of the fiber tips used in this study.

$45^{\circ}$ with respect to the evaporated beam and rotated during the entire process. This represents, to our knowledge, the first experimental implementation of an optically triggered nanoscopic fiber tip electron source.

We have found that unassisted electron field emission is possible from our pulled fiber tips with various thin layer metal coatings. Tungsten $(\mathrm{W})$, chromium $(\mathrm{Cr})$, platinum $(\mathrm{Pt})$, and gold $\mathrm{(Au}$ ) coatings in the 10-40 nm thickness range have resulted in cold field emission for applied extraction voltages in the $1-3 \mathrm{kV}$ range for an anode a few $\mathrm{mm}$ away from the tip. For the present study, we have used tungsten (W) as the electron emitter material, which was coated on a $100 \mathrm{~nm}$ diameter pulled fiber tip. The fiber tip was mounted at a distance of $10 \mathrm{~mm}$ in front of a microchannel plate imaging detector (MCP) inside of our vacuum chamber as shown in Fig. 2. Laser coupling to the fiber was achieved outside the chamber using a fiber feedthrough for the air-vacuum interface. The fiber tip was kept grounded and an extraction voltage in the $1-2.5 \mathrm{kV}$ range was applied on the front MCP plate. The emitted beam was imaged using a phosphor behind the MCP, while the corresponding emission current at the tip was measured using a highly sensitive ammeter (Agilent B2902A).

\section{RESULTS AND DISCUSSION}

Electron field emission was detected for an extraction voltage of about $1.1 \mathrm{kV}$ at $10^{-9}$ mbar background pressure. In order to estimate the electric field present at the fiber apex, we fit our I-V characteristics using a zero-temperature

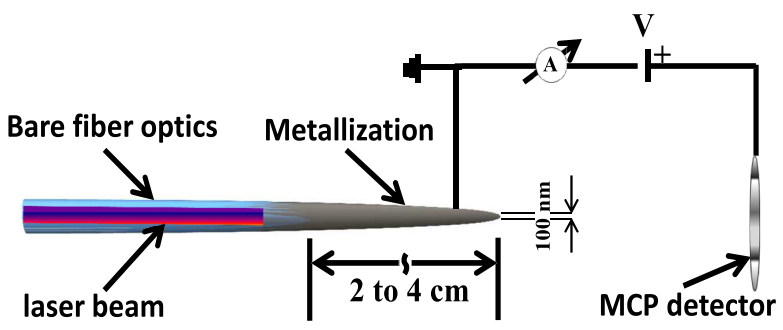

FIG. 2. (Color online) Description of the experimental setup and electrical circuit. standard Fowler-Nordheim (FN) equation for an imagecharge potential, ${ }^{28}$ which predicts a behavior as $I \propto F^{2} \exp \left(-v b\left(\phi^{3 / 2} / F\right)\right)$, where the prefactor $b$ in the FN exponent is given by $6.83 \mathrm{eV}^{-3 / 2} \mathrm{~V} / \mathrm{nm}$. The dimensionless constant $v$ is close to unity for our parameters, ${ }^{29}$ and it is therefore disregarded in our treatment. To analyze the I-V behavior found in our experiment and shown in Fig. 3, we assume the extraction voltage $V$ to be proportional to the field at the apex $F$, and hence fit our data with a FN equation of the form $I=A_{\mathrm{FN}}\left(V / B_{\mathrm{FN}}\right)^{2} \exp \left(-\left(B_{\mathrm{FN}} / V\right)\right)$. By equating $-b \phi^{3 / 2} / F=B_{\mathrm{FN}} / V$ and using our fit result $B_{\mathrm{FN}} \approx 3.85 \times 10^{4} \mathrm{~V}$, we infer an electric field at the apex of $F \approx 2.3 \mathrm{GV} / \mathrm{m}$ at an extraction voltage of $V=1300 \mathrm{~V}$ and assuming the bulk material work function of tungsten to be $\phi=4.6 \mathrm{eV}$. At this field, the Schottky-reduced tunneling barrier $^{30}$ (effective work function) is expected to be $2.79 \mathrm{eV}$. We are aware that using the bulk work function may not be accurate for predicting absolute currents, but still should provide a reasonable approximation for the present application.

As a proof-of-principle experiment, we investigated the effect on the electron emission of up to $2 \mathrm{~mW}$ of continuous $405 \mathrm{~nm}$ diode laser light applied to the fiber input, in particular, with respect to the transient switch-on behavior. In order to gain more insight on what to expect from our measurements, we measured the far field optical transmission of an uncoated fiber tip similar to the ones used in our experiments. For this purpose, a low-numerical-aperture $(\mathrm{NA}<0.01)$ lens system was used to form an image of the tip on a low-noise CCD camera. After taking and analyzing images from various polar angles with respect to the optical axis, we could reconstruct the angular emission pattern largely unhampered by background signal. The emission pattern does not overlap with the tip image for most polar angles. To infer the absolute value of the transmitted power, we used a previously obtained calibration of the camera signal and the known aperture area of the lens. The optical power transmission efficiency of the fiber was inferred to be on the order of $10^{-7}$. We note here that, for this measurement, polarization was ignored, which may lead to an azimuthal dependence of the far-field emission pattern. On the

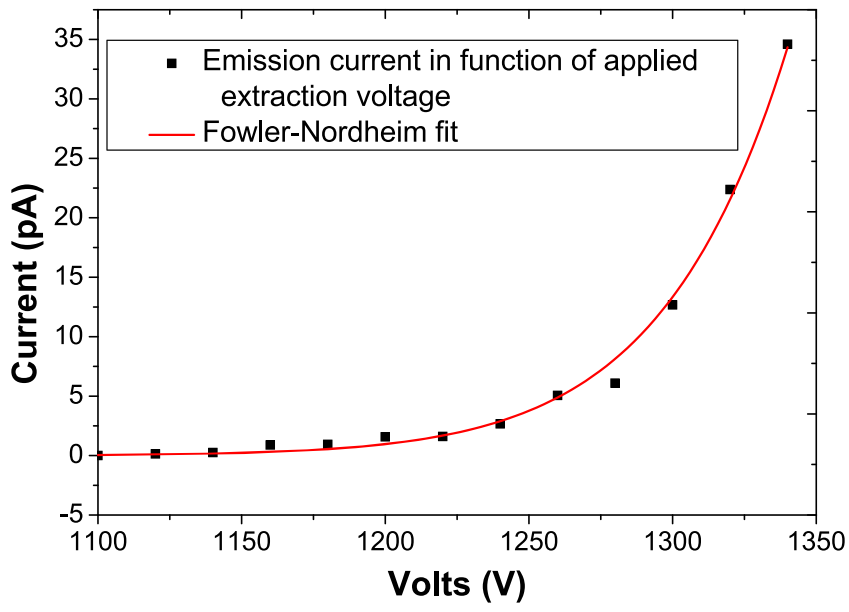

FIG. 3. (Color online) Field emission curve (squares) and the corresponding Fowler-Nordheim fit (solid line). 


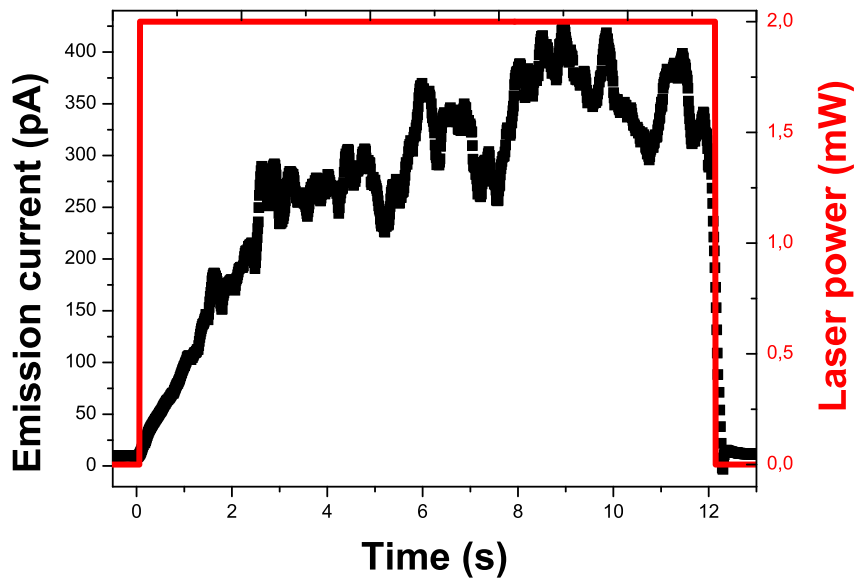

FIG. 4. (Color online) Response of electron emission to application of laser for an unconditioned tip, and in the presence of no field-emission current (black squares); the applied $405 \mathrm{~nm} \mathrm{CW}$ laser power (red solid line).

other hand, the momentum distribution of the mode coming out of the fiber tip is broad and could stretch into evanescent momentum regions. Therefore, the classic case of a flat photocathode with a plane wave propagating along an axis that is normal to its surface cannot apply here.

Given the low optical transmission, we can argue that, while single-photon photofield emission should be allowed at the used wavelength due to the Schottky lowering discussed above, the low incident peak power and transmission of the subwavelength fiber tip would make such a process extremely weak. For example, for the given conditions in order to get an emission current of $10 \mathrm{pA}$, which is close to the noise limit of our measurement, the quantum efficiency of our metal coating would have to be on the order of $1.5 \times 10^{-1}$, which is much larger than what is expected for metals. Despite these considerations, our measurements clearly reveal an enhanced field-emission current in the presence of light as shown in Figs. 4 and 5.

The switch-on electron emission time, when applying the CW light, proves to be very sensitive to the fabrication procedure, particularly the angle under which the tip is rotating in the deposition machine, the coating material, and the thickness of the coating in the $10-40 \mathrm{~nm}$ range that we investigated. Also, for similar tips, we measured different electron emission transients, depending on the time that the tip was conditioned and on the base field-emission current at which the laser

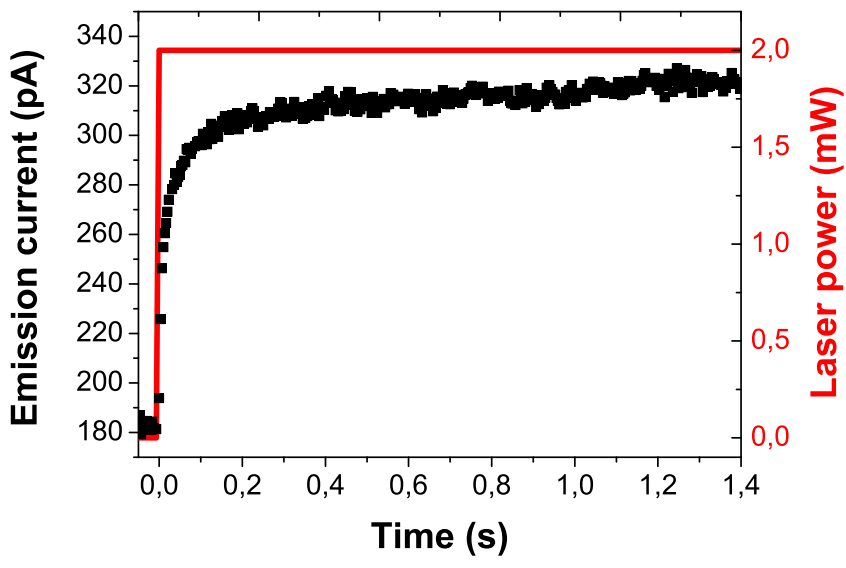

FIG. 5. (Color online) Response of electron emission to application of laser for the conditioned tip, and in the presence of a field-emission current of about $180 \mathrm{pA}$ (black squares); the applied $405 \mathrm{~nm} \mathrm{CW}$ laser power (red solid line).

triggering was applied. Figure 4 shows the electron emission switch-on time for a $10 \mathrm{~nm}$ W coated tip (deposited under $45^{\circ}$ rotation angle) before any conditioning and in the presence of no emission due to the applied electric filed. The structure of this fiber tip approximates the sketch represented in Fig. 1. Under these conditions, the time required for the emitted electron current to reach a steady state was measured to be on the order of a few seconds as it can be seen in Fig. 4. The complete recovery of the emitted current to the initial value (before the laser pulse) strongly suggests that no change on the tip structure was encountered during these measurements.

On the other hand, Fig. 5 represents the switch-on time for an identically fabricated tip, after 2 weeks of $1-1.5 \mathrm{~h}$ of measurements per day. In this case, the CW light was applied when the tip was already field-emitting a current of about $180 \mathrm{pA}$. For these conditions, an electron emission transient with a half rise time of only approximately $10 \mathrm{~ms}$ was measured. This is significantly faster, suggesting that structural changes have occurred at the tip.

Still, this excludes photofield emission as the predominant emission process, which should follow the laser diode rise time instantaneously. This fact leads us to the assumption that a thermal enhancement of the field emission current is present, where the fiber nanotip is heated by the incident laser. The emission current increase is based on changes induced in the population of electron states inside the $\mathrm{W}$
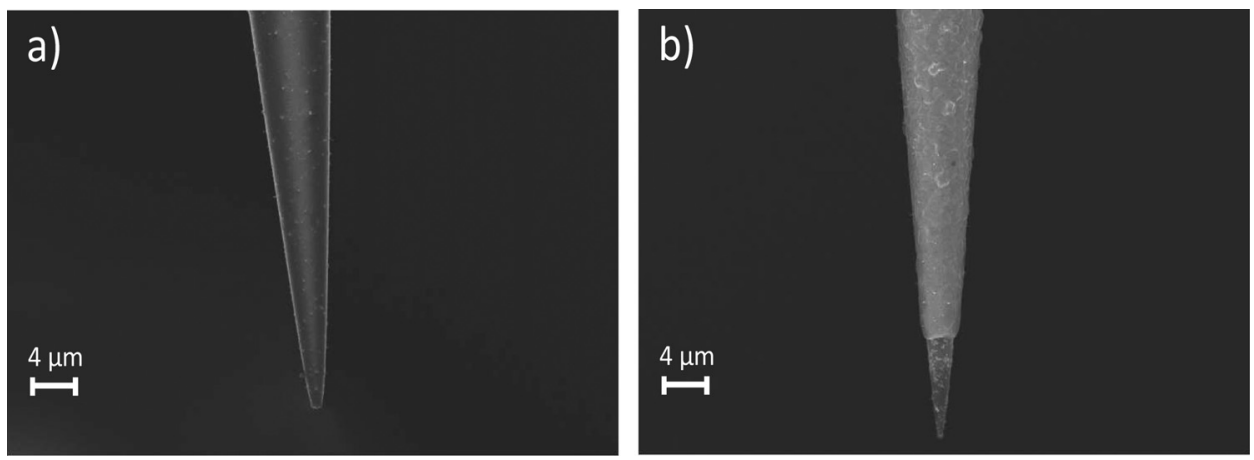

FIG. 6. SEM image of a fiber tip before the experiment (a) and after measurements and conditioning (b). 


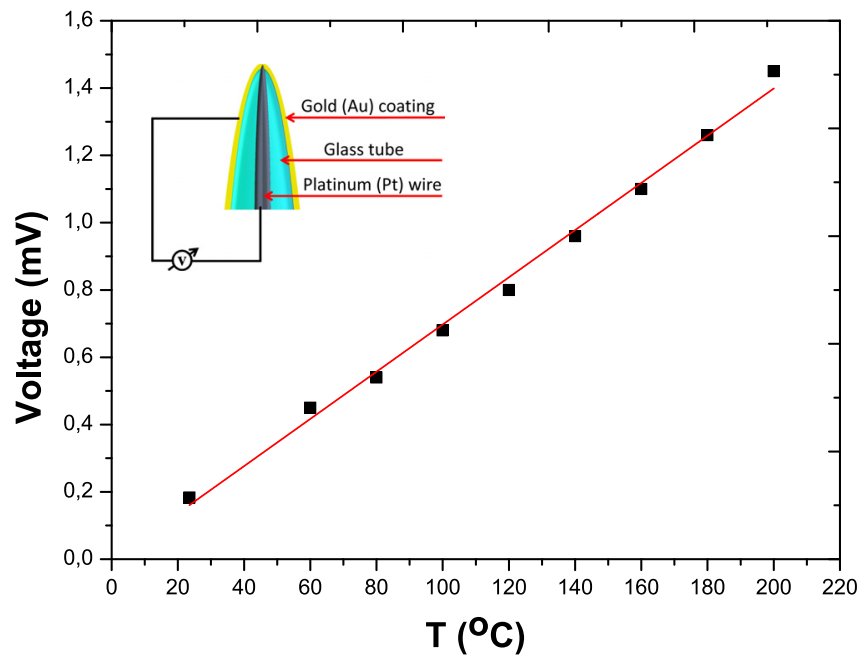

FIg. 7. (Color online) Thermocouple voltage calibration and, in the inset, the thermocouple sketch and the basic circuit are shown.

layer. At a higher temperature, states above the Fermi level become more populated, enhancing tunneling through the barrier which is lowered by Schottky-type image charge potential $^{30}$ in the presence of the high electric field. As discussed in various theory publications ${ }^{31-33}$ and also shown experimentally in Ref. 34, this can lead to significant current increase even far away from the thermionic (Richardson) regime, where electron states with energies above the barrier become populated thermally. The change of the electron emission transient could be due to structural modifications of the tip during conditioning. This explanation is strongly supported by our SEM images of the tips before and postconditioning. Figure 6 represents a similar fiber tip before the experiment (a) and after the experiment (b) when the tip became very unstable, and was not emitting anymore. The image clearly shows that ablation of the metallization has occurred at the fiber tip, likely as a result of the application of the extraction field or the $405 \mathrm{~nm}$ light, or a combination of both. This could lead to changes in the heat capacity and heat conduction properties of the emission region, which will influence the local thermal dynamics and the

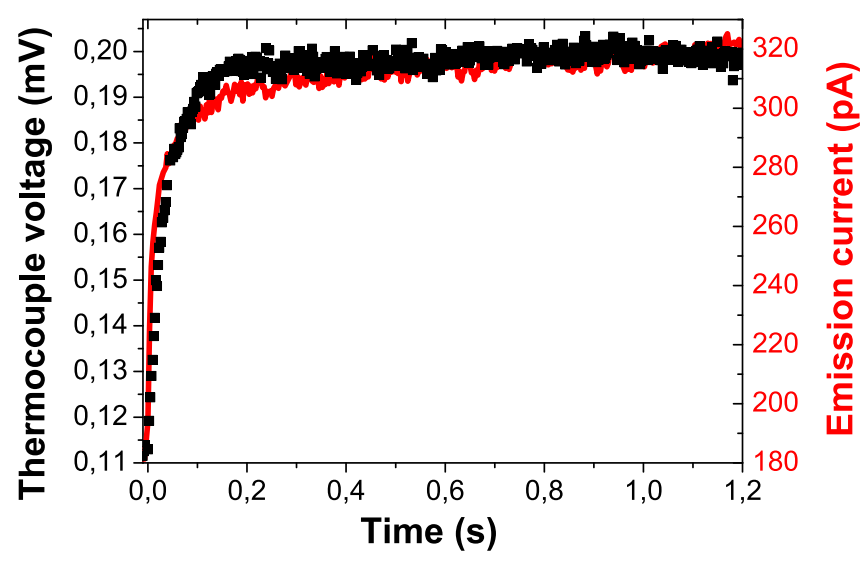

FIG. 8. (Color online) In-air temperature switch-on curve after triggering the fiber tip with the $405 \mathrm{~nm} \mathrm{CW}$ laser (black squares) and the fast optically enhanced electron emission switch-on curve from Fig. 5 (red solid line). concomitant emission current transient. Accurate modeling of the relationship between the electron emission rise time and the structural changes of the tips would however involve a detailed finite element analysis, which is beyond the scope of the current work.

In order to validate the hypothesis of thermally enhanced field emission, we used the $10 \mathrm{~nm} \mathrm{~W}$ coated fiber tip that exhibited the fastest switch-on time (Fig. 5) to measure the in-air temporal thermal transient that is triggered by the incident light. For this purpose, we used a nanometric thermocouple probe based on a standard $\mathrm{Pt} / \mathrm{Au}$ junction. The probe was made from a metal electrode purchased from Nanonics imaging Ltd. The latter consisted of platinum (Pt) wire inside of a glass tube, which was pulled at one end to form a $1 \mu \mathrm{m}$ diameter tip. We coated this structure with a gold $(\mathrm{Au})$ layer on the outside as shown in the inset of Fig. 7. The Seebeck coefficient of the device was inferred to be about $7 \mu \mathrm{V} / \mathrm{K}$ from the calibration curve presented in Fig. 7. This value is consistent with values reported in literature for a $\mathrm{Pt} /$ Au junction. ${ }^{35}$ The measurement of the thermal response of the NSOM tip was achieved by touching the two tips using a precision three-axis translation stage and measuring the voltage across the junction.

In Fig. 8, the result of the in-air temperature switch-on time measurement is shown, and a corresponding temperature increase at the tip of about $18 \mathrm{~K}$ can be inferred. We also investigated the effect of the base vacuum on this temperature increase, and no significant difference was observed for a base pressure down to $10^{-2}$ mbar. The measured thermal half-rise time $t_{1 / 2} \cong 21 \mathrm{~ms}$ is shown to be very similar to the corresponding value of the emission current switch-on time observed for the same tip. Therefore, we consider this match of thermal transients and the electron emission transient after conditioning as proof corroborating the photothermal emission hypothesis. We note that the measured temperature increase with the nanometric thermocouple is not sufficient to fully explain the enhanced current. This is the temperature rise at the contact point which is relatively far away from the very apex where the temperature could be much higher and is not directly measureable.

\section{CONCLUSIONS AND OUTLOOK}

We have presented the first experimental results on a fiber tip-based electron source with a $100 \mathrm{~nm}$ apex size, triggered by a $\mathrm{CW}$ laser at $405 \mathrm{~nm}$ under the action of an extraction electric field. Our measurements show that field emission occurs as expected for a range of coating materials, and that the application of $405 \mathrm{CW}$ light clearly enhances the emission current, with a temporal profile indicating a predominant thermal effect. While direct photoemission triggered from the fiber has not been shown yet, this new approach opens up the field of a new class of electron emitters which hold the advantages of sharp emitters and, at the same time, eliminate the difficulties of optical element alignments. Also, we successfully fabricated a nanometric thermocouple probe which showed to have a Seebeck coefficient comparable to the literature value, and a fast response time. Due to 


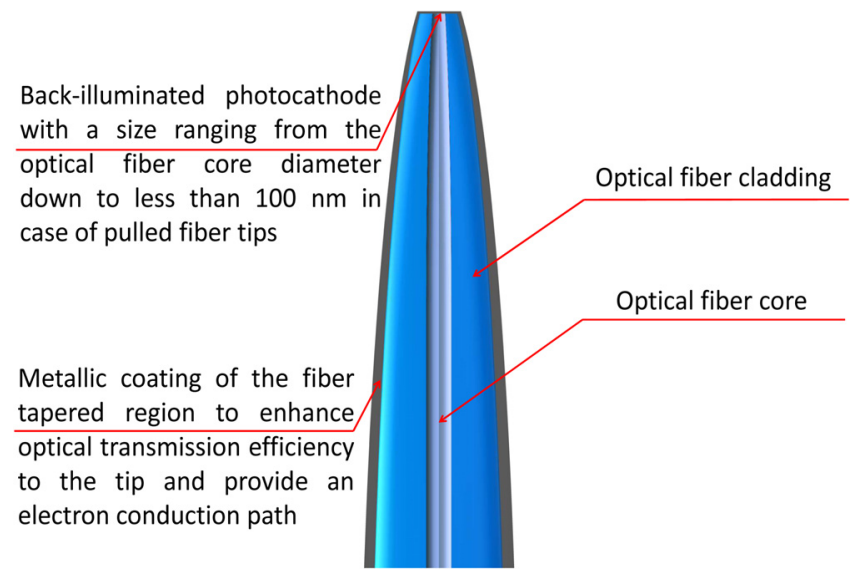

FIG. 9. (Color online) Sketch of an optical fiber tip where the size of the open fiber core aperture can be adjusted by fiber cutting techniques or laser ablation.

its dimensions, the thermocouple probe could be conveniently used for in-situ temperature measurement and stabilization during electron emission experiments.

In future work, we plan to investigate the effect of shorter wavelengths in the ultraviolet, and shorter pulse durations down to femtoseconds, on the electron emission process. These approaches should reduce the thermal effect and give larger photon transmission efficiencies at the tip and a higher quantum efficiency of the emitter metal, facilitating direct, potentially ultrafast photoemission. Also, the thickness and material of the coating layer play a very important role with respect to the achievable electron emission current, and we intend to explore the effect of the variation of these parameters more thoroughly in future work. Furthermore, we found that the optical transmission efficiency of the uncoated tips can be systematically increased up to more than $90 \%$ by cutting off the pulled tip to larger aperture sizes as represented in Fig. 9. Using fiber cutting techniques or laser ablation, the open aperture size can be varied up to the size of the initial optical fiber core of several $\mu \mathrm{m}$. Therefore, coated fiber cathodes are able to cover an entire range of electron emission regimes, where the predominant emission process could be adjusted from field emission to thermal or photoemission, or any combination of these processes. ${ }^{36}$

\section{ACKNOWLEDGMENTS}

The authors would like to thank Miriam Barthelmess and Henry Chapman's group from Center for Free Electron Laser Science (CFEL) for giving us the opportunity of using their e-beam deposition machine to coat the NSOM fiber tips and the thermocouple. The authors are also grateful to Carsten Thönnißen and Hans-Peter Oepen fom the
University of Hamburg for recording the SEM images of the fiber tips. This work was funded by the Max Planck Society.

${ }^{1}$ R. J. Dwayne Miller, Annu. Rev. Phys. Chem. 65, 583 (2014).

${ }^{2}$ R. J. Dwayne Miller, Science 343, 1108 (2014).

${ }^{3}$ J. B. Siwick, J. R. Dwyer, R. E. Jordan, and R. J. Dwayne Miller, Science 302, 1382 (2003).

${ }^{4}$ A. H. Zewail, Annu. Rev. Phys. Chem. 57, 65 (2006).

${ }^{5}$ W. E. King, G. H. Campbell, A. Frank, B. Reed, J. F. Schmerge, B. Siwick, B. C. Stuart, and P. M. Weber, J. Appl. Phys. 97, 111101 (2005).

${ }^{6}$ L. Piazza, D. J. Masiel, T. LaGrange, B. W. Reed, B. Barwick, and F. Carbone, Chem. Phys. 423, 79 (2013).

${ }^{7}$ C. A. Brau, Nucl. Instrum. Methods Phys. Res., A 407, 1 (1998).

${ }^{8}$ C. M. Tang, A. C. Ting, and T. Swyden, Nucl. Instrum. Methods Phys. Res., A 318, 353 (1992).

${ }^{9}$ F. O. Kirchner, S. Lahme, F. Krausz, and P. Baum, New J. Phys. 15, 63021 (2013).

${ }^{10}$ M. J. G. Lee, Phys. Rev. Lett. 30, 1193 (1973).

${ }^{11}$ P. Hommelhoff, Y. Sortais, A. Aghajani-Talesh, and M. Kasevich, Phys. Rev. Lett. 96, 77401 (2006).

${ }^{12}$ P. Hommelhoff, C. Kealhofer, and M. Kasevich, Phys. Rev. Lett. 97, 247402 (2006).

${ }^{13}$ C. Ropers, D. R. Solli, C. P. Schulz, C. Lienau, and T. Elsaesser, Phys. Rev. Lett. 98, 43907 (2007).

${ }^{14}$ J. Hoffrogge, J. P. Stein, M. Krüger, M. Förster, J. Hammer, D. Ehberger, P. Baum, and P. Hommelhoff, J. Appl. Phys. 115, 94506 (2014).

${ }^{15}$ R. Ganter et al., Phys. Rev. Lett. 100, 064801 (2008).

${ }^{16} \mathrm{H}$. Yanagisawa, C. Hafner, P. Doná, M. Klöckner, D. Leuenberger, T. Greber, M. Hengsberger, and J. Osterwalder, Phys. Rev. Lett. 103, 257603 (2009).

${ }^{17}$ H. Yanagisawa, M. Hengsberger, D. Leuenberger, M. Klöckner, C. Hafner, T. Greber, and J. Osterwalder, Phys. Rev. Lett. 107, 087601 (2011).

${ }^{18}$ B. Barwick, C. Corder, J. Strohaber, N. Chandler-Smith, C. Uiterwaal, and H. Batelaan, New J. Phys. 9, 142 (2007).

${ }^{19}$ A. Paarmann et al., J. Appl. Phys. 112, 113109 (2012).

${ }^{20}$ G. Sciani and R. J. Dwayne Miller, Rep. Prog. Phys. 74, 096101 (2011).

${ }^{21}$ S. Tsujino, P. Beaud, E. Kirk, T. Vogel, H. Sehr, J. Gobrecht, and A. Wrulich, Appl. Phys. Lett. 92, 193501 (2008).

${ }^{22}$ A. Mustonen, P. Beaud, E. Kirk, T. Feurer, and S. Tsujino, Appl. Phys. Lett. 99, 103504 (2011).

${ }^{23}$ P. Helfenstein, A. Mustonen, and S. Tsujino, Appl. Phys. Express 6, 114301 (2013).

${ }^{24}$ A. Mustonen, V. Guzenko, C. Spreu, T. Feurer, and S. Tsujino, Nanotechnology 25, 085203 (2014).

${ }^{25}$ S. Tsujino, M. Paraliev, E. Kirk, C. Gough, S. Ivkovic, and H. H. Braun, Phys. Plasmas 18, 64502 (2011).

${ }^{26}$ A. L. Lereu, A. Passian, and Ph. Dumas, Int. J. Nanotechnol. 9, 488 (2012).

${ }^{27}$ T. Vecchione, U. Weierstall, C. Edgcombe, and J. C. H. Spence, Microsc. Microanal. 12, 146 (2006).

${ }^{28}$ R. Forbes, Appl. Phys. Lett. 89, 113122 (2006).

${ }^{29} \mathrm{R}$. Forbes and K. Jensen, Ultramicroscopy 89, 17 (2001).

${ }^{30} \mathrm{R}$. Fowler and L. Nordheim, Proc. R. Soc. London 119, 173 (1928).

${ }^{31}$ K. Jensen, J. Vac. Sci. Technol. B 21, 1528 (2003).

${ }^{32}$ E. Murphy and R. Good, Phys. Rev. 102, 1464 (1956).

${ }^{33}$ K. Jensen and M. Cahay, Appl. Phys. Lett. 88, 154105 (2006).

${ }^{34}$ X. He, J. Scharer, J. Booske, and S. Sengele, J. Vac. Sci. Technol., B 26, 770 (2008).

${ }^{35}$ Clemens J. M. Lasance, "The Seebeck Coefficient," accessed November 11, 2014, http://www.electronics-cooling.com/2006/11/theseebeck-coefficient.

${ }^{36}$ K. L. Jensen, J. Appl. Phys. 102, 024911 (2007). 